\title{
Construction and Validation of MESPA - Self Rating Scale (Mental health, Emotional intelligence, Self esteem, Personality and Achievement motivation Self Rating Scale)
}

\author{
*Shyny T. Y \\ Ph.D (Psychology) Bharathiar University, Coimbatore \\ Corresponding Author: Shyny T. Y
}

\begin{abstract}
NEO FFI are limited to a few studies because It is constructed to measure only 5 factors of personality like Neuroticism, , Extraversion, Openness to experience, Agreeableness and Conscientiousness. But for advance study and research multidimensional factors of human beings should be measured. So there is a need for preparing new rating scale which can measure 5 aspects of human beings like Mental health, Emotional intelligence, Self esteem, Personality and Achievement motivation. This rating scale has a great significance in the study of correlating parenting styles with all these 5 dimensions of adolescents and also important in clinical and research settings with children suffering from poor self esteem, mental health disorders and personality problems .
\end{abstract}

Keywords: Mental health, Emotional intelligence, Self esteem, Personality, Achievement motivation

Date of Submission: 05-07-2017

Date of acceptance: 17-07-2017

\section{INTRODUCTION}

In the mid- to late-1990s, Costa and McCrae came to understand that some items on the NEO PI-R were outdated or too difficult for many test-takers to understand. Research also began to show that the NEO PI-R had the potential to be used with adolescents and children as young as 10 . The possibility of using the NEO with young people led Costa and McCrae in 2002 to administer the NEO PI-R to over 1,900 high school students. The research identified 48 "problem" items that reflected either participant difficulties with item wording and/or low corrected item total correlations (CITCs). Alternative items were developed to replace the "problem" items; the revised instrument was administered to new samples The NEO PI-R and a revised version of the instrument were administered to 500 adolescents, 635 adults, and 449 middle school age children (12-13 year old) For both the adolescent and adult sample, the item/facet correlations were higher for the revised version of the NEO than for NEO PI-R. In addition, the internal consistency, factor structure, and tests of convergent and discriminant validity suggested that the revised version could be used with middle school children. Of the original 48 "problem" items, 37 were improved in terms of clarity and/or CITC. In 2005, Costa and McCrae published the latest version of the NEO Inventories, NEO PI-3. The new version included revisions of 37 items. With the creation of the NEO PI-3, Costa and McCrae intended to make the inventory accessible to a wider portion of the population. The improved readability of the NEO PI-3 compared to the NEO PI-R allowed the newer measure to be used with younger populations and adults with lower educational levels. Additionally, with the replacement of the 37 items, the psychometric properties of the NEO PI-3 were slightly improved over the NEO PI-R. In addition to increasing the readability of the NEO PI, the NEO PI-3 added a glossary of less familiar terms to aid in administration. In this study NEOFFI with 60 item is used for the correlative study with MESPA self rating scale. NEOFFI is used to measure only five factors of personality . But newly constructed MESPA can measure five dimensions of a person like Mental health, Emotional intelligence, Self esteem, Personality and Achievement motivation.

\section{Review Of Literature}

The five most important dimensions of personality are conscientiousness, emotional stability, agreeableness, extraversion, and openness. The five-factor representation was originally described by Tupes and Christal (1961), on the basis of reanalysis of various data sets using bipolar variables constructed by Cattell (1951) and Goldb1 992). Cattell was the first to develop a method for improving the factor analytic pursuit of basic personality traits by commencing with a complete field of personality traits, called the trait sphere (Cattell 
1951). ). Psychometric Properties of the NEO Five-Factor Inventory Costa and McCrae's $(1989,1992)$ NEO Personality Inventory (NEO PI), Revised NEO Personality Inventory (NEO PI-R), and NEO Five-Factor Inventory (NEO FFI) were developed with the aim of assessing the five domains of the FFM: (1) neuroticism $(\mathrm{N})$, the tendency to experience negative emotions and psychological distress in response to stressors; (2) extraversion (E), the degree of sociability, positive emotionality, and general activity; (3) openness to experience (O), levels of curiosity, independent judgment, and conservativeness; (4) agreeableness (A), altruistic, sympathetic, and cooperative tendencies; and (5) conscientiousness 1 Boston University, Boston, MA, USA Corresponding Author: Anthony J. Rosellini, Center for Anxiety and Related Disorders, Department of Psychology, Boston University, 648 Beacon Street, 6th Floor, Boston, MA 02215-2013, USA Email: ajrosell@bu.edu 28 Assessment 18(1) (C), one's level of self-control in planning and organization. The five domains are hypothesized to be relatively orthogonal to one another. The NEO inventories are composed of descriptive statements (e.g., "I am not a worrier," "I really enjoy talking to people") rated on a 5-point Likerttype scale $(1=$ strongly disagree to $5=$ strongly agree $)$. The NEO PI and PI-R consist of 180 and 240 items, respectively, and may be used to compute five domain (i.e., N, E, O, A, and C) and 30 facet (six sub factors for each of the five domains) scores. In contrast, the NEO FFI contains 60 items and may be used to derive only the five domain scores (12 items per domain). NEO FFI items were selected from the NEO PI items that demonstrated the strongest correlations with their respective domain factor score, regardless of the item's intended facet (i.e., the 30 NEO PI facets are not equally represented by NEO FFI items). Each of the five domains of the NEO FFI has been found to possess adequate internal consistency and temporal stability $(\mathrm{a}=.68$ to .86 , Costa \& McCrae, 1992; $r=.86$ to .90 , Robins, Fraley, Roberts, \& Trzesniewski, 2001). Despite favourable reliability estimates, principal component analysis (PCA), exploratory factor analysis (EFA), and confirmatory factor analysis (CFA) examinations of the NEO FFI have produced mixed findings. For instance, Egan, Deary, and Austin (2000) evaluated the NEO FFI in a large nonclinical sample using PCA and EFA with varimax and oblique rotations. Although the anticipated five-factor structure was generally supported (i.e., the majority of items loaded adequately onto their expected factor), a handful of items were found to have (a) salient cross-loadings (e.g., items with loadings $\geq .30$ on more than one factor), and/or (b) non salient primary factor loadings (e.g., items with no loadings $\geq .30$ ). Such findings are consistent with prior PCA and EFA examinations of the NEO FFI in nonclinical samples (e.g., Holden \& Fekken, 1994; Parker \& Stumpf, 1998) and have served as a catalyst for studies attempting to resolve these undesirable results. For instance, McCrae and Costa (2004) responded to Egan et al. (2000) by evaluating each NEO FFI item and replacing those that consistently performed poorly in PCA and EFA. Weak items were identified by quantifying the extent of empirical support for each item with a thorough literature review (e.g., calculating how many studies found certain items to have loadings < .30). In total, 14 items were identified and replaced with other items from their respective NEO PI-R domain. Unfortunately, these revisions resulted in only trivial psychometric improvements, leading the authors to conclude that published version of NEO FFI was sufficient. Fewer studies have evaluated the NEO FFI with CFA. Although Egan et al. (2000) conducted a CFA to replicate the solution obtained with PCA/EFA, they did not report goodness-of-fit statistics. Studies that have considered CFA model fit have failed to support the conjectured latent structure of the NEO FFI. For instance, Schmitz, Hartkamp, Baldini, Rollnik, and Tress's (2001) CFA of the NEO FFI in a sample of German outpatients with psychosomatic complaints found that two-, four-, and five-factor models failed to result in adequate fit (e.g., goodness-of-fit index $=.82$ to .84 , root mean square residual $=.12$ to .16 ). Moreover, CFA inter factor correlations failed to support hypotheses about the orthogonal nature of the NEO domains (e.g., N and E r = .46). Marsh et al. (in press) recently obtained similar results using CFA in a large nonclinical sample (i.e., poor model fit, high inter factor correlations). Although CFA examinations of the NEO FFI are limited to a few studies, these findings are consistent with results obtained in CFA studies of the NEO PI and NEO PI-R (e.g., non significant factor loadings and poor goodness-of-fit statistics; Church \& Burke, 1994; Parker, Bagby, \& Summerfeldt, 1993). In addition to criticisms about the lack of psychometric support for the NEO FFI (e.g., Egan et al., 2000; Parker \& Stumpf, 1998; Schmitz et al., 2001), such findings have led researchers to question the adequacy of CFA in the study of personality structure (see Aluja, Garcia, Garcia, \& Seisdedos, 2005; Church \& Burke, 1994; McCrae, Zonderman, Costa, Bond, \& Paunonen, 1996; Parker et al., 1993; Vassend \& Skrondal, 1997). For instance, McCrae et al. (1996) argued that "there is no theoretical reason why traits should not have meaningful loadings on three, four, or five factors" (p. 553). Likewise, others have hypothesized that the congeneric model used in CFA (i.e., specification of each indicator to load onto a single latent factor) is overly restrictive, as personality indicators are prone to have salient secondary loadings unless factors are defined by only a small number of nearly synonymous items (Church \& Burke, 1994). Marsh et al. (2009) also discuss how CFA models that fix cross-loadings to zero may inflate NEO inter factor correlations to appear non orthogonal. Much of this discussion reflects a fundamental difference in how CFA and EFA attempt to obtain simple structure (i.e., the most interpretable solution). In EFA with two or more factors, factor rotation is needed to obtain simple structure because the factor-loading matrix is fully saturated (i.e., all indicators are freely 
estimated). Conversely, factor rotation is unnecessary in CFA because simple structure is obtained by fixing most (if not all) item-factor cross-loadings to zero. Accordingly, the increased parsimony of CFA models (i.e., model over identification) allows for model specifications not possible in the EFA framework (e.g., freely estimating indicator error co-variances). Along these lines, a good-fitting measurement model is needed prior to examining structural (i.e., regressive) paths between latent variables, thus making CFA an important prelude to structural equation modelling. Exploratory structural equation modelling (ESEM) is a recently developed methodology that combines the techniques of EFA and CFA (see Asparouhov \& Muthén, 2009). ESEM is unique in that it may be Rosellini and Brown 29 used to simultaneously examine EFA and CFA measurement models and generate parameters estimates according to either framework. For example, ESEM may be used to freely estimate the relationships between all observed and latent variables, implement orthogonal and oblique factor rotations, specify correlated errors, calculate standard errors and goodness-of-fit statistics, and regress endogenous latent variables on exogenous latent variables. The advancement of ESEM has allowed researchers to examine the properties of the NEO FFI in novel ways. Marsh et al. (in press) was the first study to use ESEM to evaluate the NEO FFI. The data were modeled with and without a priori specification of 57 correlated errors corresponding to NEO FFI item pairs derived from the same NEO PI facets (e.g., correlated error was specified between Items 1 and 21 because both load on the Anxiety facet of the N domain of the NEO PI). Although ESEM without correlated errors provided better model fit than CFA, fit statistics were still generally below prevailing standards of acceptable fit (e.g., Tucker-Lewis index $[\mathrm{TLI}]=.82$; comparative fit index $[\mathrm{CFI}]=.85$ ). In contrast, ESEM with correlated errors resulted in marginally acceptable fit $(\mathrm{TLI}=.89$; CFI $=.91$; root mean square error of approximation [RMSEA] $=.03$ ), by far the most promising model fit ever obtained for the NEO FFI. ESEM also resulted in weaker inter factor correlations than CFA, which is more consistent with FFM theory (i.e., the five domains are hypothesized to be orthogonal). Although the goodness-of-fit statistics obtained from the ESEM models were modest relative to proposed "cutoffs" (e.g., TLI and CFI near or greater than .95; Hu \& Bentler, 1999), others have contended that these guidelines may be overly restrictive (e.g., Beauducel \& Whittmann, 2005; Marsh, Hau, \& Grayson, 2005; Marsh, Hau, \& Wen, 2004). In particular, Marsh et al. (2005) recommend that psychometric evaluations of longer questionnaires (e.g., 50 or more items, five or more factors) should not use model fit guidelines with excessive strictness. Moreover, Marsh et al. (in press) conclude that traditional CFA models are not appropriate for the NEO FFI and that ESEM should be used in its place to utilize the benefits of confirmatory models (e.g., adjustment for measurement error). Although the findings of Marsh et al.'s (in press) ESEM support the factor structure of the NEO FFI in normative samples. But no study has yet existed for measure Mental health, Emotional intelligence, Self esteem, Personality and Achievement motivation at a time.

\section{Method}

64 Participants in this study were of the adolescents between the age group of 12 to 18. Mean age of participants was 15. The gender of the participant was unequal. MESPA Self Rating sale with 45 items were employed here. Reliability for MESPA Self Rating scale is completed. Split half reliability and item analysis is find out by using participants of this study. Validity was examined through a comparison with the NEOFFI. 5 Personal aspects like Mental health, Emotional intelligence, Self esteem, Personality and Achievement motivation are embedded here in a random order. 1,4,10,14,19,24,28,32,37,41 are mental health items. $8,17,26,35,44$ are items of emotional intelligence. 7,16,25,34,23 included under self esteem. 2,11,20,29,38 are personality extraversion items. $3,12,21,30,39$ are personality openness items. $5,13,22,31,40$ are personality agreeableness items. 6,15,23,33,42 are personality conscientiousness items. 9,18,27,36,45 are items of achievement motivation.

\section{Research Hypothesis}

1.There will be consistent personal dimensions across ages as reported by Parent subjects of adolescents with age group twelve, fifteen $\&$ eighteen.

2.There will be relatively high positive correlation between NEOFFI Extraversion, Openness, Agreeableness, Conscientiousness and MESPA Extraversion, Openness, Agreeableness, Conscientiousness.

3.The Item analysis of Reliability Coefficient will be greater than .70 for the MESPA SELF RAING SCALE

\section{Results}

Table 1

\begin{tabular}{|l|l|l|}
\hline Reliability-NEW & Cronbach's Alpha & Nof Items \\
\hline MESPA & 0.990 & 45 \\
\hline Reliability-OLD & Cronbach's Alpha & Nof Items \\
\hline NEOFFI & 0.961 & 60 \\
\hline
\end{tabular}

TABLE 1 shows reliability is .99 for the MESPA and .96 forNEOFFI. So we can say this newly constructed MESPA Self Rating Scale has very strong internal consistency. 
Table-2 Means, Standard Deviation and F value for Age- NEOFFI

\begin{tabular}{|c|c|c|c|c|c|c|}
\hline Variable & Age & $N$ & Mean & $\begin{array}{c}\text { Standar } \\
\text { d Deviation }\end{array}$ & $F$ & $p$ value \\
\hline \multirow{3}{*}{ Neurotic } & 12 years & 22 & 24.82 & 15.09 & & \\
\hline & 15 years & 22 & 25.82 & 18.41 & 0.018 & 0.982 \\
\hline & 18 years & 20 & 25.30 & 19.11 & & \\
\hline \multirow{3}{*}{ Extraversion } & 12 years & 22 & 38.36 & 20.96 & & \\
\hline & 15 years & 22 & 38.77 & 21.61 & 0.150 & 0.861 \\
\hline & 18 years & 20 & 41.70 & 21.36 & & \\
\hline \multirow{3}{*}{ Openness } & 12 years & 22 & 35.36 & 21.05 & & \\
\hline & 15 years & 22 & 38.09 & 19.84 & 0.340 & 0.713 \\
\hline & 18 years & 20 & 40.30 & 17.07 & & \\
\hline \multirow{3}{*}{ Agreeableness } & 12 years & 22 & 26.05 & 17.75 & & \\
\hline & 15 years & 22 & 27.27 & 18.69 & 0.176 & 0.839 \\
\hline & 18 years & 20 & 24.15 & 14.42 & & \\
\hline \multirow{3}{*}{ Conscientiousn } & 12 years & 22 & 26.05 & 17.76 & & \\
\hline & 15 years & 22 & 27.36 & 18.51 & 0.311 & 0.734 \\
\hline & 18 years & 20 & 23.30 & 13.98 & & \\
\hline
\end{tabular}

Tabl-3 Means, Standard Deviation and F value for Age-MESPA

\begin{tabular}{|c|c|c|c|c|c|c|}
\hline Variable & Age & $N$ & Mean & $\begin{array}{c}\text { Standar } \\
\text { d Deviation }\end{array}$ & $\boldsymbol{F}$ & p value \\
\hline \multirow{3}{*}{$\begin{array}{l}\text { Mental } \\
\text { health }\end{array}$} & 12 years & 22 & 16.55 & 11.04 & & \\
\hline & 15 years & 22 & 16.77 & 10.91 & 0.210 & 0.811 \\
\hline & 18 years & 20 & 18.60 & 11.55 & & \\
\hline \multirow{3}{*}{$\begin{array}{l}\text { Emotional } \\
\text { intelligence }\end{array}$} & 12 years & 22 & 8.41 & 5.59 & & \\
\hline & 15 years & 22 & 8.50 & 5.71 & 0.041 & 0.960 \\
\hline & 18 years & 20 & 8.90 & 6.36 & & \\
\hline \multirow{3}{*}{ Self esteem } & 12 years & 22 & 8.23 & 5.73 & & \\
\hline & 15 years & 22 & 8.77 & 5.55 & 0.256 & 0.775 \\
\hline & 18 years & 20 & 9.45 & 5.30 & & \\
\hline \multirow{3}{*}{ Extraversion } & 12 years & 22 & 7.82 & 5.95 & & \\
\hline & 15 years & 22 & 8.59 & 5.76 & 0.421 & 0.658 \\
\hline & 18 years & 20 & 9.45 & 5.52 & & \\
\hline \multirow{3}{*}{ Openness } & 12 years & 22 & 3.91 & 4.36 & & \\
\hline & 15 years & 22 & 5.41 & 5.50 & 0.981 & 0.381 \\
\hline & 18 years & 20 & 3.45 & 4.35 & & \\
\hline \multirow{3}{*}{ Agreeablene } & 12 years & 22 & 3.86 & 5.05 & & \\
\hline & 15 years & 22 & 5.18 & 5.76 & 0.721 & 0.490 \\
\hline & 18 years & 20 & 3.35 & 4.44 & & \\
\hline \multirow{3}{*}{$\begin{array}{l}\text { Conscientio } \\
\text { usness }\end{array}$} & 12 years & 22 & 8.27 & 5.58 & & \\
\hline & 15 years & 22 & 8.82 & 5.58 & 0.261 & 0.771 \\
\hline & 18 years & 20 & 9.55 & 6.06 & & \\
\hline \multirow{3}{*}{$\begin{array}{l}\text { Achievemen } \\
\text { t motivation }\end{array}$} & 12 years & 22 & 16.59 & 12.04 & & \\
\hline & 15 years & 22 & 16.36 & 12.16 & 0.020 & 0.980 \\
\hline & 18 years & 20 & 17.10 & 12.38 & & \\
\hline
\end{tabular}

In table 2 and table 3 Age group was considered to be the independent variable, which included three age groups as (a) 12 years; (b) 15 years; and (c) 18 year. The results of the ANOVA test depicted in Table 2 reveals that statistical value is greater than 0.05 for all the variables. So we conclude that the mean score of different variables does not differs with age.

Table-4

\begin{tabular}{|c|l|c|c|c|c|}
\hline Correlation-TOTAL & Correlation & $\begin{array}{c}\text { Lowe } \\
\mathrm{r} \text { bound }\end{array}$ & $\begin{array}{c}\text { Uppe } \\
\mathrm{r} \text { bound }\end{array}$ & $\mathrm{Z}$ & $\mathrm{p}$ \\
\hline MES-NEO & 0.899 & 0.883 & 0.915 & 16.163 & $<0.001$ \\
\hline Age = 12 years & Correlation & $\begin{array}{l}\text { Lower } \\
\text { bound }\end{array}$ & $\begin{array}{l}\text { Upper } \\
\text { bound }\end{array}$ & $\mathrm{Z}$ & $\mathrm{p}$ \\
\hline MES-NEO & 0.944 & 0.928 & 0.960 & 12.795 & $<0.001$ \\
\hline Age = 15 years & Correlation & $\begin{array}{l}\text { Lower } \\
\text { bound }\end{array}$ & $\begin{array}{l}\text { Upper } \\
\text { bound }\end{array}$ & $Z$ & $p$ \\
\hline MES-NEO & 0.908 & 0.883 & 0.933 & 9.692 & $<0.001$ \\
\hline Age = 18 years & Correlation & $\begin{array}{l}\text { Lower } \\
\text { bound }\end{array}$ & $\begin{array}{l}\text { Upper } \\
\text { bound }\end{array}$ & $\mathrm{Z}$ & $\mathrm{p}$ \\
\hline MES-NEO & 0.842 & 0.798 & 0.886 & 6.622 & $<0.001$ \\
\hline
\end{tabular}

A positive correlation exist for the variables of new and old scales as in these case the correlation coefficient has value greater than 0.5 and $\mathrm{p}$ value less than 0.05 .

Table-5 
Construction and Validation of Mespa - Self Rating Scale (Mental health, Emotional intelligence, Self

\begin{tabular}{|c|c|c|c|c|c|}
\hline $\begin{array}{c}\text { The correlation between old and new -total } \\
\text { data }\end{array}$ & $\begin{array}{c}\text { Correlation } \\
\text { coefficient }\end{array}$ & $\begin{array}{c}\text { Lowe } \\
\text { r bound }\end{array}$ & $\begin{array}{c}\text { Uppe } \\
\text { r bound }\end{array}$ & Z & p \\
\hline Extraversion-Old and Extraversion-New & $0.895^{*}$ & 0.878 & 0.912 & 15.799 & $<0.001$ \\
\hline Openness-Old and Openness-New & 0.486 & 0.422 & 0.550 & 4.379 & $<0.001$ \\
\hline Agreeableness-Old and Agreeableness-New & $0.896^{*}$ & 0.879 & 0.913 & 15.888 & $<0.001$ \\
\hline $\begin{array}{c}\text { Conscientiousness-Old and Conscientiousness- } \\
\text { New }\end{array}$ & $0.606^{*}$ & 0.553 & 0.659 & 5.999 & $<0.001$ \\
\hline
\end{tabular}

\section{*Significant correlation}

Pearson Correlation is used to identify the relationship between old an new questionnaires and the result is exhibited in. We can conclude that correlation is significant. Here Table5 point out this significant correlation.

Table-6

\begin{tabular}{|c|c|c|c|c|c|}
\hline Age $=12$ years & Correlation & Lower bound & Upper bound & $\mathrm{Z}$ & $\mathrm{p}$ \\
\hline Extraversion-Old and Extraversion-New & 0.906 & $0.880^{*}$ & 0.932 & 9.572 & $<0.001$ \\
\hline Openness-Old and Openness-New & 0.496 & 0.388 & 0.604 & 2.555 & 0.018 \\
\hline Agreeableness-Old and Agreeableness-New & 0.833 & $0.789^{*}$ & 0.877 & 6.733 & $<0.001$ \\
\hline $\begin{array}{l}\text { Conscientiousness-Old } \\
\text { Conscientiousness-New }\end{array}$ & 0.713 & $0.642 *$ & 0.784 & 4.548 & $<0.001$ \\
\hline Age $=15$ years & Correlation & Lower bound & Upper bound & $\mathrm{Z}$ & $\mathrm{p}$ \\
\hline Extraversion-Old and Extraversion-New & 0.890 & $0.860^{*}$ & 0.920 & 8.729 & $<0.001$ \\
\hline Openness-Old and Openness-New & 0.558 & 0.459 & 0.657 & 3.007 & 0.006 \\
\hline Agreeableness-Old and Agreeableness-New & 0.943 & $0.927 *$ & 0.959 & 12.672 & $<0.001$ \\
\hline $\begin{array}{l}\text { Conscientiousness-Old } \\
\text { Conscientiousness-New }\end{array}$ & 0.680 & $0.603^{*}$ & 0.757 & 4.148 & $<0.001$ \\
\hline Age $=18$ years & Correlation & Lower bound & Upper bound & $\mathrm{Z}$ & $\mathrm{p}$ \\
\hline Extraversion-Old and Extraversion-New & 0.894 & $0.864 *$ & 0.924 & 8.465 & $<0.001$ \\
\hline Openness-Old and Openness-New & 0.422 & 0.298 & 0.546 & 1.975 & 0.062 \\
\hline Agreeableness-Old and Agreeableness-New & 0.918 & $0.894 *$ & 0.942 & 9.821 & $<0.001$ \\
\hline $\begin{array}{cc}\text { Conscientiousness-Old } & \text { and } \\
\text { Conscientiousness-New } & \\
\end{array}$ & 0.439 & 0.317 & 0.561 & 2.073 & 0.051 \\
\hline
\end{tabular}

\section{*Significant correlation}

For all variables other than openness and agreeableness correlation coefficient is less than 0.5 and $p$ value is less than 0.05 . So we can say correlation is significant.

Table-7

\begin{tabular}{|c|c|c|c|c|c|c|}
\hline Variables & group & $N$ & Mean & Std. Deviation & $z$ & $p$ value \\
\hline \multirow{2}{*}{ MES1 } & Low & 16 & 0.81 & 0.40 & \multirow{2}{*}{$\begin{array}{r}- \\
21.706\end{array}$} & \multirow{2}{*}{$<0.001$} \\
\hline & High & 16 & 3.00 & 0.00 & & \\
\hline \multirow{2}{*}{ MES2 } & Low & 16 & 0.75 & 0.45 & \multirow{2}{*}{$\begin{array}{r}- \\
20.125\end{array}$} & \multirow{2}{*}{$<0.001$} \\
\hline & High & 16 & 3.00 & 0.00 & & \\
\hline \multirow{2}{*}{ MES3 } & Low & 16 & 0.44 & 0.51 & \multirow{2}{*}{$\begin{array}{r}- \\
20.006\end{array}$} & \multirow{2}{*}{$<0.001$} \\
\hline & High & 16 & 3.00 & 0.00 & & \\
\hline \multirow{2}{*}{ MES4 } & Low & 16 & 0.44 & 0.51 & \multirow{2}{*}{$\begin{array}{r}- \\
20.006\end{array}$} & \multirow{2}{*}{$<0.001$} \\
\hline & High & 16 & 3.00 & 0.00 & & \\
\hline \multirow{2}{*}{ MES5 } & Low & 16 & 0.00 & 0.00 & \multirow{2}{*}{$\begin{array}{r}- \\
11.662 \\
\end{array}$} & \multirow{2}{*}{$<0.001$} \\
\hline & High & 16 & 2.31 & 0.79 & & \\
\hline \multirow{2}{*}{ MES6 } & Low & 16 & 0.00 & 0.00 & \multirow{2}{*}{$\begin{array}{r}- \\
13.024 \\
\end{array}$} & \multirow{2}{*}{$<0.001$} \\
\hline & High & 16 & 2.63 & 0.81 & & \\
\hline \multirow{2}{*}{ MES7 } & Low & 16 & 0.00 & 0.00 & & \\
\hline & High & 16 & 3.00 & 0.00 & & \\
\hline \multirow{2}{*}{ MES8 } & Low & 16 & 0.00 & 0.00 & & \\
\hline & High & 16 & 3.00 & 0.00 & & \\
\hline \multirow{2}{*}{ MES9 } & Low & 16 & 0.00 & 0.00 & & \\
\hline & High & 16 & 3.00 & 0.00 & & \\
\hline \multirow{2}{*}{ MES10 } & Low & 16 & 0.31 & 0.48 & \multirow{2}{*}{$\begin{array}{r}- \\
22.456 \\
\end{array}$} & \multirow{2}{*}{$<0.001$} \\
\hline & High & 16 & 3.00 & 0.00 & & \\
\hline \multirow{2}{*}{ MES11 } & Low & 16 & 0.38 & 0.50 & \multirow{2}{*}{$\begin{array}{r}- \\
21.000 \\
\end{array}$} & \multirow{2}{*}{$<0.001$} \\
\hline & High & 16 & 3.00 & 0.00 & & \\
\hline \multirow{2}{*}{ MES12 } & Low & 16 & 0.38 & 0.50 & & $<0 \Omega 01$ \\
\hline & High & 16 & 3.00 & 0.00 & 21.000 & -0.001 \\
\hline & Low & 16 & 0.00 & 0.00 & - & \\
\hline MES13 & High & 16 & 2.38 & 0.81 & 11.783 & $<0.001$ \\
\hline & Low & 16 & 0.06 & 0.25 & - & \\
\hline MES14 & High & 16 & 3.00 & 0.00 & 47.000 & $<0.001$ \\
\hline MES15 & Low & 16 & 0.00 & 0.00 & - & $<0001$ \\
\hline IVIESIS & High & 16 & 2.50 & 0.82 & 12.247 & -0.001 \\
\hline MFS16 & Low & 16 & 0.00 & 0.00 & & \\
\hline MESIO & High & 16 & 3.00 & 0.00 & & \\
\hline & Low & 16 & 0.44 & 0.51 & - & \\
\hline MESI/ & High & 16 & 3.00 & 0.00 & 20.006 & $<0.001$ \\
\hline
\end{tabular}




\begin{tabular}{|c|c|c|c|c|c|c|}
\hline \multirow{2}{*}{ MES18 } & Low & 16 & 0.44 & 0.51 & \multirow{2}{*}{$\begin{array}{r}- \\
20.006 \\
\end{array}$} & \multirow{2}{*}{$<0.001$} \\
\hline & High & 16 & 3.00 & 0.00 & & \\
\hline \multirow{2}{*}{ MES19 } & Low & 16 & 0.25 & 0.45 & \multirow{2}{*}{$\begin{array}{r}- \\
24.597\end{array}$} & \multirow{2}{*}{$<0.001$} \\
\hline & High & 16 & 3.00 & 0.00 & & \\
\hline \multirow{2}{*}{ MES20 } & Low & 16 & 0.13 & 0.34 & \multirow{2}{*}{$\begin{array}{r}- \\
33.669\end{array}$} & \multirow{2}{*}{$<0.001$} \\
\hline & High & 16 & 3.00 & 0.00 & & \\
\hline \multirow{2}{*}{ MES21 } & Low & 16 & 0.00 & 0.00 & & \\
\hline & High & 16 & 3.00 & 0.00 & & \\
\hline \multirow{2}{*}{ MES22 } & Low & 16 & 0.00 & 0.00 & \multirow{2}{*}{$\begin{array}{r}- \\
12.247\end{array}$} & \multirow{2}{*}{$<0.001$} \\
\hline & High & 16 & 2.50 & 0.82 & & \\
\hline \multirow{2}{*}{ MES23 } & Low & 16 & 0.06 & 0.25 & \multirow{4}{*}{$6.504^{-}$} & \multirow{2}{*}{$<0.001$} \\
\hline & High & 16 & 1.81 & 1.05 & & \\
\hline \multirow{2}{*}{ MES24 } & Low & 16 & 0.00 & 0.00 & & \\
\hline & High & 16 & 3.00 & 0.00 & & \\
\hline \multirow{2}{*}{ MES25 } & Low & 16 & 0.25 & 0.45 & - & $<0001$ \\
\hline & High & 16 & 3.00 & 0.00 & 24.597 & $<0.001$ \\
\hline MES26 & Low & 16 & 0.25 & 0.45 & - & $<0001$ \\
\hline $\operatorname{IVICS} \angle 0$ & High & 16 & 3.00 & 0.00 & 24.597 & -0.001 \\
\hline MES27 & Low & 16 & 0.38 & 0.50 & - & $<0001$ \\
\hline IVIESLI & High & 16 & 3.00 & 0.00 & 21.000 & -0.001 \\
\hline MFS28 & Low & 16 & 0.00 & 0.00 & & \\
\hline IVIES 20 & High & 16 & 3.00 & 0.00 & & \\
\hline MFS 20 & Low & 16 & 0.00 & 0.00 & & \\
\hline IVICS29 & High & 16 & 3.00 & 0.00 & & \\
\hline MES30 & Low & 16 & 0.00 & 0.00 & & \\
\hline IVIESSU & High & 16 & 3.00 & 0.00 & & \\
\hline & Low & 16 & 0.00 & 0.00 & - & \\
\hline NIESSI & High & 16 & 2.69 & 0.70 & 15.267 & $<0.001$ \\
\hline & Low & 16 & 0.13 & 0.34 & - & \\
\hline IVIESS2 & High & 16 & 3.00 & 0.00 & 33.669 & $<0.001$ \\
\hline & Low & 16 & 0.00 & 0.00 & - & \\
\hline MES33 & High & 16 & 2.50 & 0.73 & 13.693 & $<0.001$ \\
\hline MES34 & Low & 16 & 0.13 & 0.34 & - & $<0001$ \\
\hline NIESS4 & High & 16 & 3.00 & 0.00 & 33.669 & $<0.001$ \\
\hline MES35 & Low & 16 & 0.00 & 0.00 & & \\
\hline IVIESSS & High & 16 & 3.00 & 0.00 & & \\
\hline MES36 & Low & 16 & 0.13 & 0.34 & - & $<0,001$ \\
\hline IVIESSO & High & 16 & 3.00 & 0.00 & 33.669 & -0.001 \\
\hline MES37 & Low & 16 & 0.00 & 0.00 & & \\
\hline IVIESSI & High & 16 & 3.00 & 0.00 & & \\
\hline MES 38 & Low & 16 & 0.19 & 0.40 & - & $<0001$ \\
\hline IVILSJo & High & 16 & 3.00 & 0.00 & 27.908 & -0.001 \\
\hline MFS39 & Low & 16 & 0.00 & 0.00 & & \\
\hline IVIES59 & High & 16 & 3.00 & 0.00 & & \\
\hline MFS40 & Low & 16 & 0.00 & 0.00 & - & $<0001$ \\
\hline IVIESU & High & 16 & 2.13 & 0.72 & 11.825 & -0.001 \\
\hline MES41 & Low & 16 & 0.00 & 0.00 & & \\
\hline MIES4I & High & 16 & 3.00 & 0.00 & & \\
\hline & Low & 16 & 0.00 & 0.00 & - & \\
\hline MIES42 & High & 16 & 2.50 & 0.82 & 12.247 & $<0.001$ \\
\hline & Low & 16 & 0.00 & 0.00 & & \\
\hline IVIES43 & High & 16 & 3.00 & 0.00 & & \\
\hline & Low & 16 & 0.00 & 0.00 & & \\
\hline IVIES44 & High & 16 & 3.00 & 0.00 & & \\
\hline & Low & 16 & 0.06 & 0.25 & - & \\
\hline MIES4J & High & 16 & 3.00 & 0.00 & 47.000 & $<0.001$ \\
\hline
\end{tabular}

Table 7 result revealed there is a strong validity for each items. P value is less than .05 ie; correlation is Significant at .001 level.

\section{Discussion}

The objective of this study is to design and construct a new measure MESPA to evaluate like Mental health, Emotional intelligence, Self esteem, Personality and Achievement motivation. Review of literature point out the use of the 5 personality dimensions instead of 5 personal dimensions. Adolescents at a particular age group of 2,15,18 is consider for the study. The 5 sub scales of the MESPA show high reliability and validity. The usual limitation of this study was small sample of 64 makes this a pilot study. Some other problem were difference in geographical areas like urban and rural. Another limitation was the Gender difference of the population. 


\section{Conclusion}

The purpose of this study was to provide a five dimensional personal self rating scale to identify their Mental health, Emotional intelligence, Self esteem, Personality and Achievement motivation .This rating scale is also important in clinical and research settings with children suffering from poor self esteem, mental health disorders sand personality problems . This study has great important in academic field as well as job selection field to find out students with high achievement motivation, emotional intelligence and good personality This research examine how these multi personal dimensions relate to adolescents of different age group 12,15 and 18. MESPA RATING SCALE shows very high reliability and validity. Item analysis shows significant correlation with NEOFFI. So we can accept MESPA as a standard tool. MESPA is a valuable gift for teachers, parents, researchers, psychologists, counsellors and psychiatrist to study their students, children, clients and patients.

\section{References}

[1] Costa P.T, \& McCrae, R.R. (1992), Normal Personality assessment in clinical practice : The NEO Personality Inventory, Psychological assessment, 4,5-13

[2] Mangala,C.V. (2009) Adjustment of students to their Home Environment, Indian journal of Psychometry and Education, 41 (1\&2), $1260-63$

[3] Mc Crae R.R., Costa P.T, Martin T.A (2005). "The NEO-PI 3: "The NEO - PI-3 : A more readable revised NEO Personality inventory". Journal of Personality assessment 84(3) : 261-270.

[4] Mccrae, R.R; \& Costa P.T (February 2004), "A contemplated revision of "A revision of the NEO Five factor Inventory". Personality and Individual differences 36 (3) : 587-596. DOI : 10.1016/SO191-8869 (03) 00118-1.

[5] Singh, T.et al. (2011) Inter active Influence of Adjustment and Gender on Academic Achievement. Psycholinguae, 41 (2), 318-325

[6] Cohen, S., \& Edwards, J. R. (in press). Personality characteristics as moderators of the relationship between stress and disorder. In R. W. J. Neufeld (Ed.), Advances in the investigation of psychological stress. New York: Wiley.

[7] Ahmed, S. M. S., Valliant, P. M., \& Swindle, D. (1985). Psychometric properties of the Coopersmith Self-Esteem Inventory. Perceptual and Motor Skills, 61, 1235-1241.

[8] Amato, P. R., \& Keith, B. (1991). Parental divorce and the well-being of children: A meta-analysis. Psychological Bulletin, 110, 26-46.

[9] American Association of University Women. (1991). Short changing girls, short changing America. Washington, DC: Author. Ammerman, M. S., \& Fryrear, J. L. (1975). Photographic enhancement of children's self-esteem. Psychology in the Schools, 12, 319-325.

[10] Arnett, J. J. (1999). Adolescent storm and stress, reconsidered. American Psychologist, 54, 317- 326

[11] Bachman, J. G., Green, S., \& Wirtanen, I. D. (1971). Youth in transition: Vol. 2. Dropping out—Problem or symptom? Ann Arbor, MI: Institute for Social Research.

[12] Bachman, J. G., \& O'Malley, P. M. (1977). Self-esteem in young men: A longitudinal analysis of the impact of educational and occupational attainment. Journal of Personality and Social Psychology, 35, 365-380.

[13] Baumeister, R. F., Smart, L., \& Boden, J. M. (1996). Relation of threatened egotism to violence and aggression: The dark side of high self-esteem. Psychological Review, 103, 5-33.

[14] Cohen, S., \& Hoberman, H. M. (1983). Positive events and social supports as buffers of life change stress. Journal of Applied Social Psychology, 13,99-125.

[15] Cohen, S., \& McKay, G. (1983). Social support, stress, and the buffering hypothesis: A theoretical analysis. In A. Baum, S. E. Taylor, \& J. Singer (Eds.), Handbook of psychology and health (Vol. 4, pp. 253- 267).

[16] Hillsdale, NJ: Erlbaum. Conway, M., \& Ross, M. (1984). Getting what you want by revising what you had. Journal of Personality and Social Psychology, 47,738- 748 .

[17] Ryan RM, and Deci EL (2001). On happiness and human potential: a review of research on hedonic and eudaimonic well-being. Annual Review of Psychology, 52, 141-166.

[18] Tennant R, Hiller L, Fishwick R, Platt S, Joseph S, Weich S, Parkinson J, Secker S, and Stewart-Brown S (2007). The WarwickEdinburgh Mental Well-being Scale (WEMWBS): development and UK validation. Health \& Quality of Life Outcomes, 5 (63), doi:10.1186/1477-7525-5-63.

[19] Tennant R, Joseph S, and Stewart-Brown S (2007). The Affecto meter 2: a measure of positive mental health in UK populations. Quality of Life Research, 16 (4), 687-695.

[20] Coyne, J. C., \& Gotlieb, 1. H. (1983). The role of cognition in depression: A critical appraisal. Psychological Bulletin, 94, 472-505.

[21] Crandall, V. J., Solomon, D., \& Kelleway, R. (1955). Expectancy statements and decision times as functions of objective probabilities and reinforcement values. Journal of Personality, 24, 192-203.

[22] Crary, W. G. (1966). Reactions to incongruent self-experiences. Journal of Consulting Psychology. 30, 246-252.

[23] Crocker, J. (1982). Biased questions in judgment of covariation studies. Personality and Social Psychology Bulletin, 8, 214-220.

[24] Cutrona, C. E. (1982). Transition to college: Loneliness and the process of social adjustment. In L. A. Peplau \& D. Perlman (Eds.), Loneliness: A sourcebook of current theory, research and therapy (pp. 291- 309). New York: Wiley.

[25] Ames, C. (1992). Classrooms: Goals, structures, and student motivation. Journal of Educational Psychology, 84, 261-271.

[26] Anderman, E., \& Maehr, M. L. (1994). Motivation and schooling in the middle grades. Review of Educational Research, 64, $287-$ 309.

[27] Austin, J., \& Vancouver, J. (1996). Goal constructs in psychology: Structure, process, and content. Psychological Bulletin, 120, $338-375$.

[28] Bandura, A. (1997). Self-efficacy: The exercise of control. New York: Freeman. Deci, E., \& Ryan, R. (1985). Intrinsic motivation and self-determination in human behavior. New York: Plenum. Dweck, C., \& Leggett, E. (1988). A social cognitive approach to motivation and personality. Psychological Review, 95, 256-273.

[29] Elliot, A. (1997). Integrating the "classic" and "contemporary" approaches to achievement motivation: A hierarchical model of approach and avoidance achievement motivation. In M.L. Maehr \& P.R. Pintrich (Eds.), Advances in motivation and achievement (Vol. 10., pp. 143-179).

[30] Greenwich, CT: JAI Press. Elliot, A., \& Church, M. (1997). A hierarchical model of approach and avoidance achievement motivation. Journal of Personality and Social Psychology, 72, 218-232.

[31] Elliot, A., \& Harackiewicz, J. (1996). Approach and avoidance achievement goals and intrinsic motivation: A mediational analysis. Journal of Personality and Social Psychology, 70, 968-980. 
[32] Darley, J. M., \& Fazio, R. H. (1980). Expectancy confirmation processes arising in the social interaction sequence. American Psychologist, 35, 867'-881. deCharms, R. (1968). Personal causation: The internal affective determinants of behavior. New York: Academic Press.

[33] Diener, C. I., \& Dweck, C. S. (1978). An analysis of learned helplessness: Continuous changes in performance, strategy, and achievement cognitions following failure. Journal of Personality and Social Psychology, 36, 451-462.

[34] Diener, C. I., \& Dweck, C.S.(1980).An analysis of learned helplessness: II. The processing of success. Journal of Personality and Social Psychology, 39, 940-952.

[35] Diener, E. (1984). Subjective well-being. Psychological Bulletin, 95, 542-575.

[36] Duval, S., \& Wicklund, R. A. (1972). A theory of objective self-awareness. New York: Academic Press.

[37] Dweck, C. S., \& Licht, B. G. (1980). Learned helplessness and intellectual achievement. In M. E. P. Seligman \& J. Garber (Eds.), Human helplessness: Theory and applications (pp. 197-222). New York: Academic Press

[38] . Eckland, B. K. (1968). Theories of mate selection. Eugenics Quarterly, 15, 71-84. Erikson, E. H. (1950). Childhood and society (2nd ed.). New York: Norton.

[39] Feather, N. T. (1966). Effects of prior success and failure on expectations of success and subsequent performance. Journal of Personality and Social Psychology, 3, 287-298.

[40] Feather, N. T. (1968). Change in confidence following success or failure as a predictor of subsequent performance. Journal of Personality and Social Psychology, 9, 38-46.

[41] Feather, N. T. (1969). Attribution of responsibility and valence of success and failure in relation to initial confidence and task performance. Journal of Personality and Social Psychology, 13, 129-144.

[42] MESPA - SELF RATING SCALE

[43] (Mental health, Emotional intelligence, Self esteem, Personality and Achievement motivation Self Rating Scale)

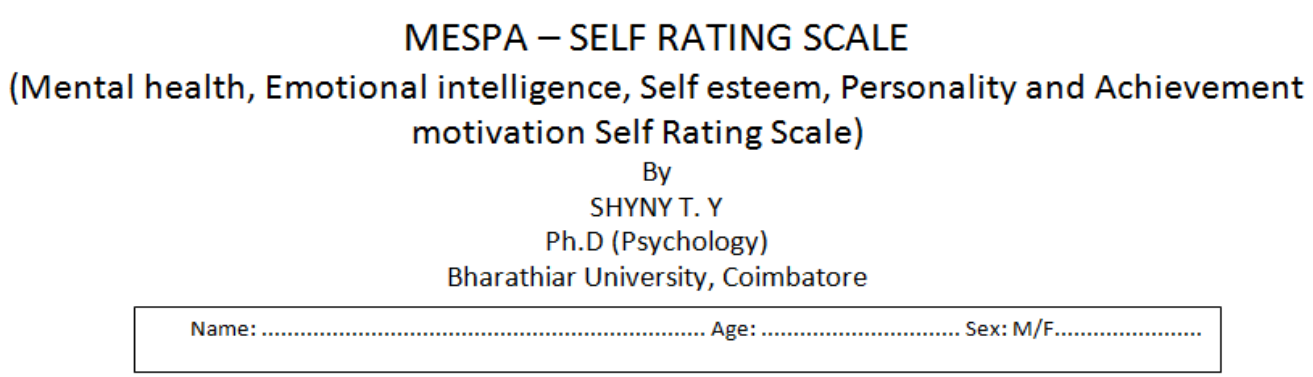

Instructions: Read each statement carefully and indicate your single response by putting a "tick" mark against it in an appropriate box

\begin{tabular}{|c|c|c|c|c|c|}
\hline $\begin{array}{l}\text { Sl } \\
\text { No }\end{array}$ & Statements & $\begin{array}{l}\text { Not true } \\
\text { at all }\end{array}$ & $\begin{array}{l}\text { Just a } \\
\text { little } \\
\text { true }\end{array}$ & $\begin{array}{l}\text { Often } \\
\text { true }\end{array}$ & $\begin{array}{l}\text { Very } \\
\text { much } \\
\text { true }\end{array}$ \\
\hline 1 & Anxiety and tension & & & & \\
\hline 2 & Laughing easily & & & & \\
\hline 3 & Trying new and foreign food & & & & \\
\hline 4 & Sad and depressed & & & & \\
\hline 5 & Courteous & & & & \\
\hline 6 & Keeping Surroundings clean and neat & & & & \\
\hline 7 & Feeling of confidence with many good qualities & & & & \\
\hline 8 & $\begin{array}{l}\text { Knowing others from their non verbal messages like voice tone, facial expression } \\
\text { etc. }\end{array}$ & & & & \\
\hline 9 & Enjoying Challenging tasks and competition & & & & \\
\hline 10 & Disturbed sleep & & & & \\
\hline 11 & Enjoying gatherings & & & & \\
\hline 12 & Fond of art and nature & & & & \\
\hline 13 & Co-operative & & & & \\
\hline 14 & Feeling of helplessness and need for others help & & & & \\
\hline 15 & Perform assigned task on time & & & & \\
\hline 16 & Ability to do things as others & & & & \\
\hline 17 & Experiencing others emotions just by looking their face. & & & & \\
\hline 18 & Starting new work just after finishing the present one. & & & & \\
\hline 19 & Feeling of inferior to others & & & & \\
\hline 20 & Active and cheerful & & & & \\
\hline 21 & Intellectual curiosity & & & & \\
\hline 22 & Thoughtful and considerate & & & & \\
\hline 23 & Working hard to achieve a goal in an orderly fashion. & & & & \\
\hline 24 & Feeling of loneliness and helplessness & & & & \\
\hline 25 & Feeling of self respect and proud & & & & \\
\hline 26 & Complimenting others and helping them for positive thinking. & & & & \\
\hline 27 & Doing things indifferent from others by using unique skills & & & & \\
\hline 28 & Digestive problem and acidity. & & & & \\
\hline 29 & Light hearted & & & & \\
\hline 30 & Abstract thinking & & & & \\
\hline 31 & Harmonious with family members and co-workers & & & & \\
\hline 32 & Asthma or other allergy problems. & & & & \\
\hline
\end{tabular}




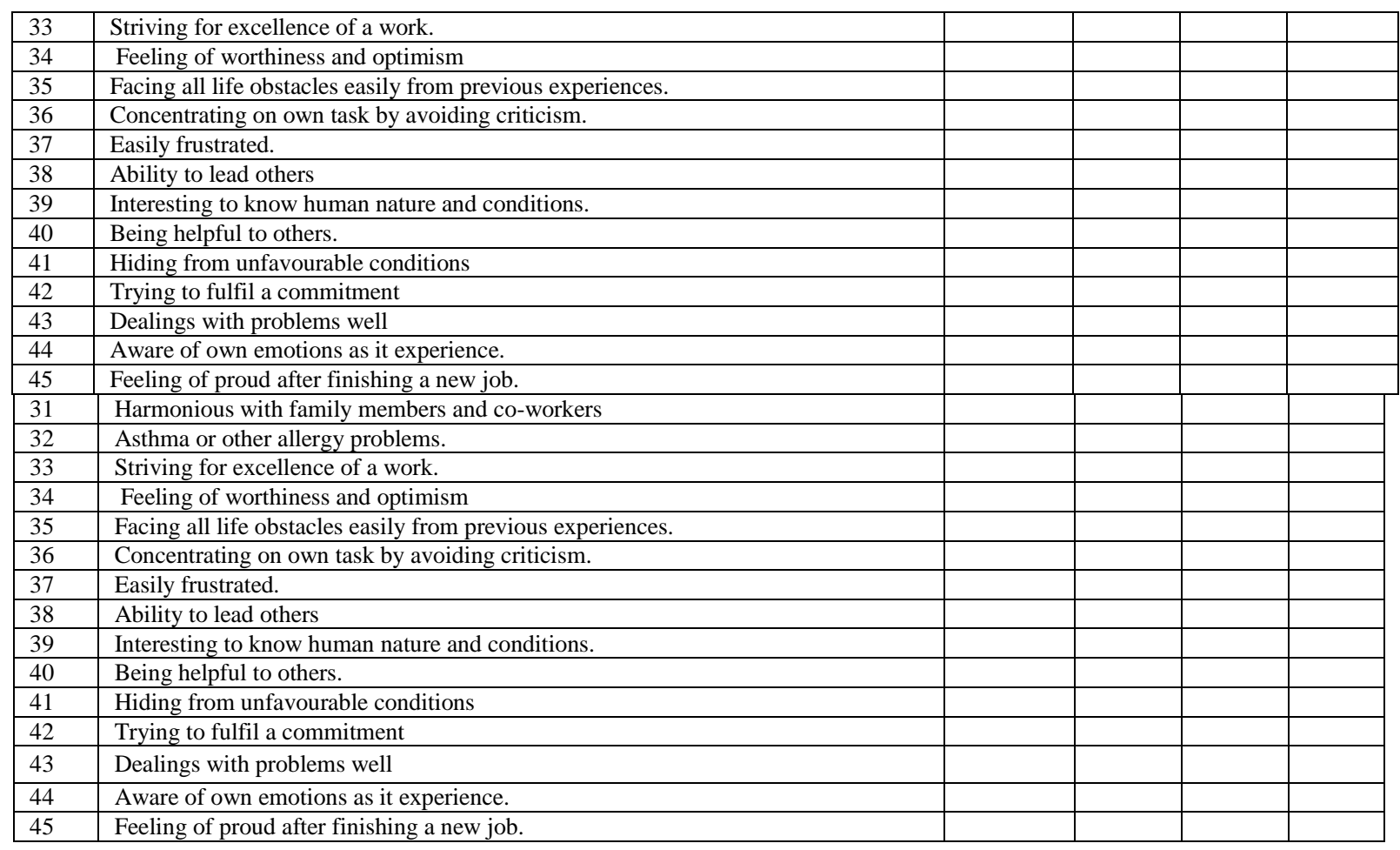

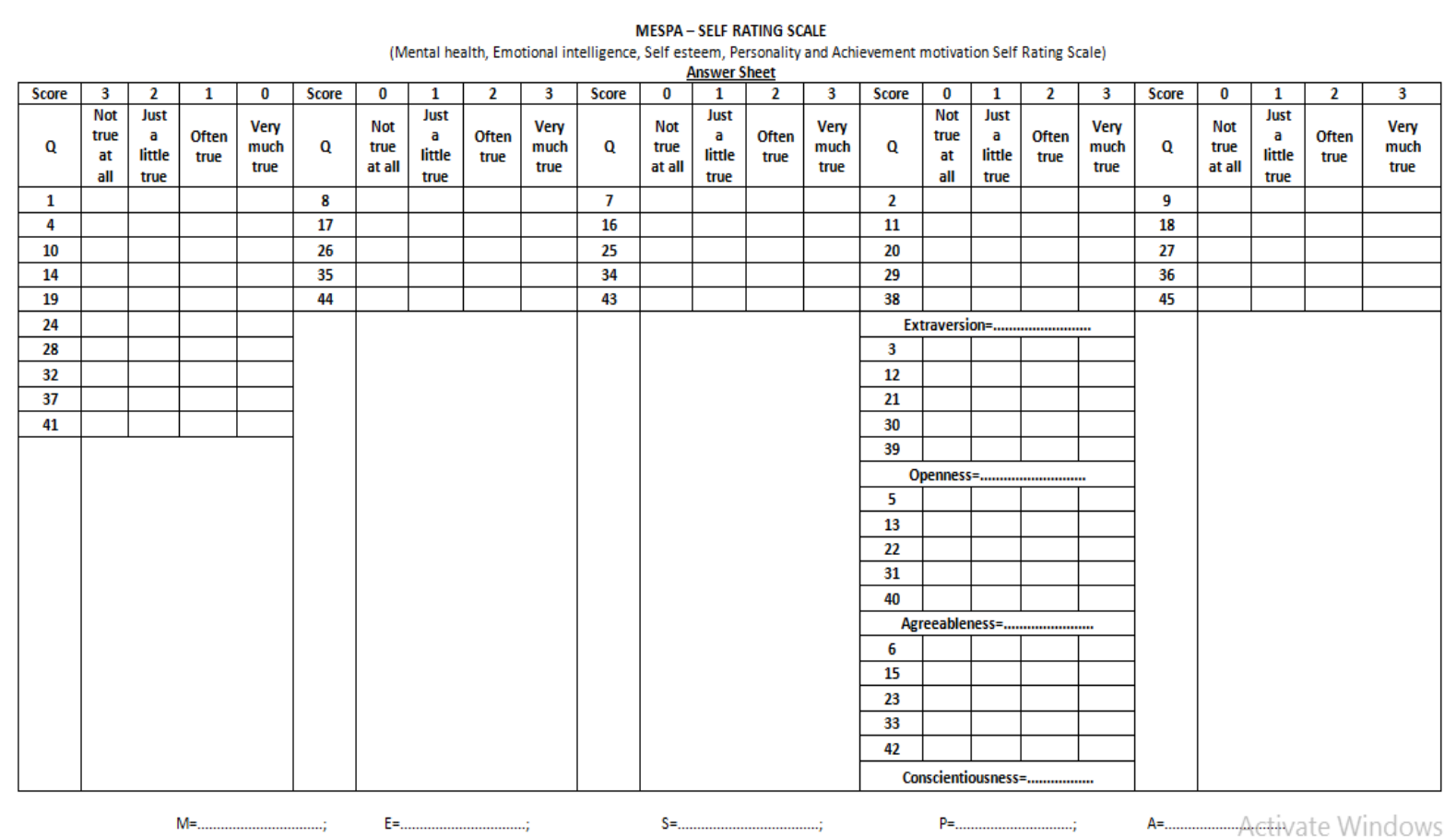

(M=Mental health, E=Emotional intelligence, $\mathrm{S}=$ Self esteem, $\mathrm{P}=$ Personality, A=Achievement motivation)

$\begin{array}{ll}\text { Have you responded to all of the statements } & \text { Yes/NQ } \\ \text { Have you entered your responses in the correct boxes } & \text { Yes/NQ } \\ \text { Have you responded accurately and honestly } & \text { Yes/NQ }\end{array}$

Name: :.......................................................................... Age: ............................... Sex: M/F.......................

Shyny T. Y. "Construction and Validation of Mespa - Self Rating Scale (Mental health, Emotional intelligence, Self esteem, Personality and Achievement motivation Self Rating Scale)." IOSR Journal of Research \& Method in Education (IOSR-JRME) 7.4 (2017): 51-59. 